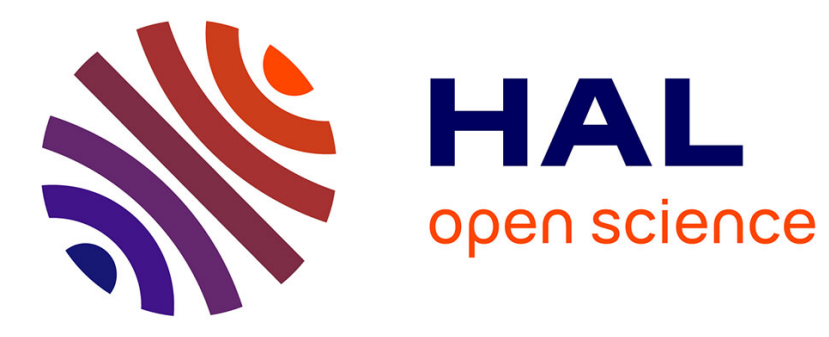

\title{
The memory of fluctuating brownian dipolar chains
}

\author{
Renaud Toussaint, Eirik Grude Flekkøy, Geir Helgesen
}

\section{To cite this version:}

Renaud Toussaint, Eirik Grude Flekkøy, Geir Helgesen. The memory of fluctuating brownian dipolar chains. Physical Review E: Statistical, Nonlinear, and Soft Matter Physics, 2006, 74 (5), pp.051405. 10.1103/PhysRevE.74.051405 . hal-00107356

\section{HAL Id: hal-00107356 https://hal.science/hal-00107356}

Submitted on 9 Nov 2018

HAL is a multi-disciplinary open access archive for the deposit and dissemination of scientific research documents, whether they are published or not. The documents may come from teaching and research institutions in France or abroad, or from public or private research centers.
L'archive ouverte pluridisciplinaire HAL, est destinée au dépôt et à la diffusion de documents scientifiques de niveau recherche, publiés ou non, émanant des établissements d'enseignement et de recherche français ou étrangers, des laboratoires publics ou privés. 


\title{
Memory of fluctuating Brownian dipolar chains
}

\author{
Renaud Toussaint, ${ }^{1, *}$ Eirik G. Flekkøy, ${ }^{2}$ and Geir Helgesen ${ }^{3}$ \\ ${ }^{1}$ Institute of Globe Physics in Strasbourg (IPGS), UMR 7516 CNRS, 5 rue Descartes, F-67084 Strasbourg Cedex, France \\ ${ }^{2}$ Department of Physics, University of Oslo, P.O. Box 1048 Blindern, NO-0316 Oslo, Norway \\ ${ }^{3}$ Institute for Energy Technology, NO-2027 Kjeller, Norway
}

(Received 19 June 2006; published 20 November 2006)

\begin{abstract}
Nonmagnetic particles in suspension in a ferrofluid act as magnetic holes when an external magnetic field is exerted: They acquire an effective dipolar moment opposing the surrounding one, which induces dipolar magnetic interactions. For a large enough imposed field and particle size, the induced interactions dominate the thermal forces, dipolar chains form. At equilibrium, these chains fluctuate under the effect of brownian noise. When the imposed magnetic field is suddenly decreased, these chains roughen dynamically. We study the time and size scaling of these fluctuations and roughening, and the relationships between the equilibrium and out of equilibrium behavior. We compare the experimental data both to Brownian dynamics simulations, and to a simple theory of semiflexible polymer chains, a generalization of the Rouse model. The scaling behavior of the experiments agree with the predictions of both theory and simulations over 5 orders of magnitudes. The roughening follows three successive regimes: The root mean square width of the chain initially evolves as $W \sim t^{1 / 2}$, then it enters a subdiffusive regime where $W \sim t^{1 / 4}$ and eventually it saturates to a level $W \sim N$, where $N$ is the number of particles in the chain. The exact prefactors as a function of the applied field, particle diameter, and temperature are also derived analytically. We also show that this phenomenon can be described equivalently as a non-Markovian diffusion process for a particle in an environment with memory effects. Within this framework, our system is shown to confirm the predictions of theories for anomalous diffusion in systems with memory.
\end{abstract}

DOI: 10.1103/PhysRevE.74.051405 PACS number(s): 82.70.Dd, 75.50.Mm, 05.40.-a, 83.10.Pp

\section{INTRODUCTION}

When micrometer sized nonmagnetic spheres are suspended in a magnetized ferrofluid they define magnetic holes that interact very much like magnetic dipoles [1-3]. These dipole interactions may be precisely controlled by the external field. In 1983, Skjeltorp introduced such a system that allowed direct observation by the use of transparent glass plates and perfectly monodisperse Ugelstad spheres [4].

A population of magnetic holes may be brought to organize in a rich variety of structures through their induced dipole interactions [2]. One of the simplest equilibrium structures that can be obtained, is a straight dipolar chain. When the particles are sufficiently small such chains exhibit lateral thermal fluctuations. We have recently described these fluctuations and shown that dipolar chains, or Brownian worms, are described by the Rouse model $[5,6]$ for polymer dynamics. Since hydrodynamic interactions between different chain segments are suppressed by the boundaries the present system in fact realizes the Rouse model more closely than any free polymer, and to our knowledge, any comparable experimental system.

The external control of the interparticle forces allows us to study the roughening of an initially straight chain. We study this dynamic process experimentally by optical microscopy, and we compare it to Brownian dynamics simulations as well as analytical results. As a main result we observe growth exponents that agree well with the predictions [5]: Initially the root-mean-square (rms) width of the chain grows

*Electronic address: renaud.toussaint@eost.u-strasbg.fr with a diffusive exponent of $1 / 2$ which subsequently crosses over to an exponent of $1 / 4$ before the chain width saturates at an asymptotic value that depends on the chain length. Analytically these exponents are derived from a discrete version of the Edwards-Wilkinson equation [7]—or equivalently, the Rouse model.

Chain fluctuations in equilibrium are closely connected to the dynamic roughening process. We show that the dynamic roughening from an initially straight chain, and the departure from an initially rough state at equilibrium, are two equivalent problems, characterized by the same Family-Vicsek scaling with same roughness and dynamic exponents. Only the prefactor of the mean square chain width is different.

Having derived the discrete Edwards-Wilkinson equation with the coefficients corresponding to our experiment, we turn to an alternative theoretical description by which we identify the dynamic roughening as a process of anomalous diffusion [8]. The long memory effects that are a prerequisite for any process of anomalous diffusion, stem from the propagation of perturbations along the chain, and we compute the observed anomalous diffusion exponent of $1 / 4$.

In general, the lateral fluctuations of dipolar chains have attracted much attention over the past two decades. The systems in which such chains have been studied include in particular magnetorheological [9-12] and electrorheological (ER) suspensions. In these systems, the dipolar moment is fixed inside the particles, in contrast to the present system where the interactions of the nonmagnetic particles only reflect the magnetization of the surrounding fluid. Much of the interest in electrorheological and magnetorheological fluids is based on their ability to form chains under application of an external field, thereby changing the rheology. Technologically these effects may allow the development of dampers, 


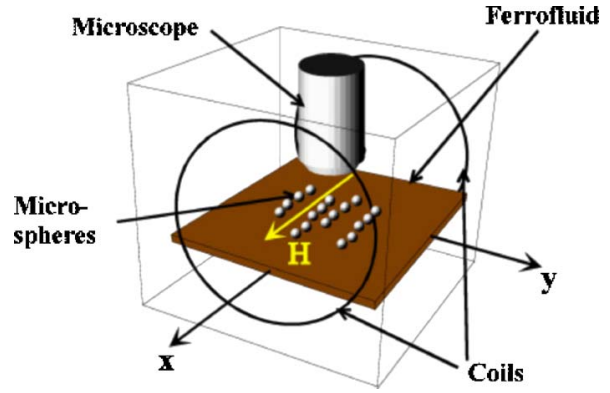

FIG. 1. (Color online) The experimental setup.

hydraulic valves, clutches or brakes [13-17]. Over time chains may aggregate laterally due to their Brownian motion. These effects are analogous to the van der Waals interaction, and have been analyzed by Halsey and Toor as well as Martin et al. [18-21]. Therefore, the precise quantification of Brownian motion of a single isolated dipolar chain is an important compound to understand the larger scale aggregation phenomena, whether this happens in magnetorheological [22-24] or electrorheological $[21,25,26]$ fluids, or in systems of magnetic holes [27,28].

The paper is organized as follows: in Sec. II, we describe the experimental system under study along with some basic theoretical considerations. In Sec. III, we derive discrete Edwards-Wilkinson equation and the dynamical scaling of a dipolar chain. In Sec. IV, we describe the numerical Brownian dynamic model designed to simulate the chain motion. In Sec. V, we compare the analytical, numerical, and experimental results for the dynamic chain roughening. In the following section, we derive analytically the connections between the out-of-equilibrium roughening problem, and the equilibrium fluctuations. Next, in Sec. VII, we derive the linear response function of the chain, and show how the dynamic scaling may be understood in the theoretical framework of diffusion in systems with memory [8]. The conclusions are eventually summarized in Sec. VIII.

\section{THEORETICAL BASIS AND THE EXPERIMENTAL SETUP}

The magnetic properties of a ferrofluid stem from suspended magnetite particles which are of nanometer size. Therefore the magnetic properties of the ferrofluid is homogeneous on the scale of the Ugelstad spheres. These spheres are of nonmagnetic polystyrene designed according to Ugelstads technique [4]. They are embedded in a kerosene-based ferrofluid [29] confined between two parallel glass plates with a $10 \mu \mathrm{m}$ separation (see Fig. 1).

Since the Ugelstad spheres are not magnetized by the external field they represent an effective dipole with a moment approximately equal to and opposite of the dipolar moment of the displaced ferrofluid. We are currently working to quantify this effective moment more precisely. However, to first approximation it may be written $\sigma=-V \bar{\chi} \mathbf{H}$ where $\chi$ is the magnetic susceptibility of the ferrofluid, $\mathbf{H}$ the magnetic field, $V$ is the holes volume and $\bar{\chi}=3 \chi /(3+2 \chi)$ is an effective susceptibility taking into account the demagnetization factor associated with the spherical shape of the particles [30]. In the absence of confining walls the magnetic interaction potential between two holes of diameter $a$, may be written $[30]$

$$
U=\frac{\mu_{f} \sigma^{2}}{4 \pi} \frac{1-3 \cos ^{2} \theta}{r^{3}},
$$

where $r$ is the distance between the centers of the spheres, with a separation vector lying at an angle $\theta$ to the external magnetic field. The ratio of the magnetic interaction energy to the thermal energy $k T$ is denoted $\lambda$, and can be estimated from the above equation as [31]

$$
\lambda=\frac{\mu_{f} \sigma^{2}}{2 \pi a^{3} k T}=\frac{\mu_{f} \bar{\chi}^{2} H^{2} \pi a^{3}}{72 k T} .
$$

When $\lambda$ exceeds 1 , the dipolar interactions lead the particles to organize in long chains parallel to the imposed field. At $\lambda \gg 1$, these chains are essentially straight and rigid, and the particles are in contact. If the external field is suddenly reduced to values that are still greater than 1 , Brownian forces from the embedding fluid leads to a dynamic roughening of the chains (see Fig. 2).

In the experiments the polystyrene spheres [4], which were of equal size, had diameters either $a=3.0$ or $4.0 \mu \mathrm{m}$. They were dispersed in a kerosene-based ferrofluid [29] of susceptibility $\chi=0.8$ and viscosity $\eta=6 \times 10^{-3} \mathrm{~Pa} \mathrm{~s}$, inside a glass cell of size $38 \mathrm{~mm} \times 8 \mathrm{~mm} \times 10 \mu \mathrm{m}$. A pair of outside coils produced a magnetic field parallel to the long axis of the cell with field strength up to $H=20$ Oe. The setup was mounted under an optical microscope using an optical magnification of $20 \times$ and having an attached video camera that recorded to a digital tape recorder at a speed of 25 frames per second. Low volume fractions $(1 \%)$ of microspheres were used and chains were grown by applying a constant field of about $H=18$ Oe parallel to the thin ferrofluid layer for about 20 minutes [28]. The cell was then searched for long chains containing typically $20-120$ spheres which were well separated from neighboring chains in order to avoid interchain interactions. The field was then reduced to a constant value $H$ in the range $2 \mathrm{Oe} \leqslant H \leqslant 10$ Oe while the motion of one long chain was recorded. The recorded video, which had a frame size of $720 \times 576$ pixels, was analyzed with a sampling rate of four frames per second. One pixel of the video image corresponded to $0.5 \mu \mathrm{m}$, and the uncertainty in particle position could be reduced to $0.2 \mu \mathrm{m}$ by utilizing the intensity profile of the pixels showing the particle. The initial roughness of the chains before reducing the field strength was less than the experimental resolution of $0.2 \mu \mathrm{m}$, and the time to reduce the magnetic field from the initial to the final value was about $1 \mathrm{~s}$, thus introducing a similar uncertainty in the starting moment of free fluctuations. During an experiment the nonmagnetic particles were centered midway between the upper and lower glass plate of the cell due to magnetic repulsion from the nonmagnetic walls (the magnetic "mirror image" effect) [2]. The experiments illustrated in Fig. 2 are challenging in part because this accuracy is needed to reveal the scaling behavior of the chains. 


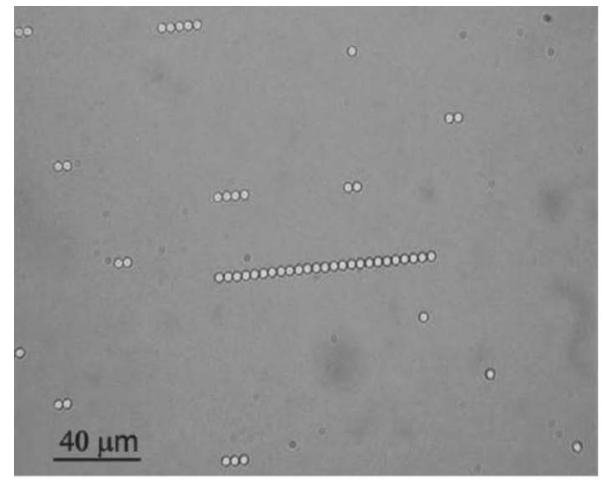

a)
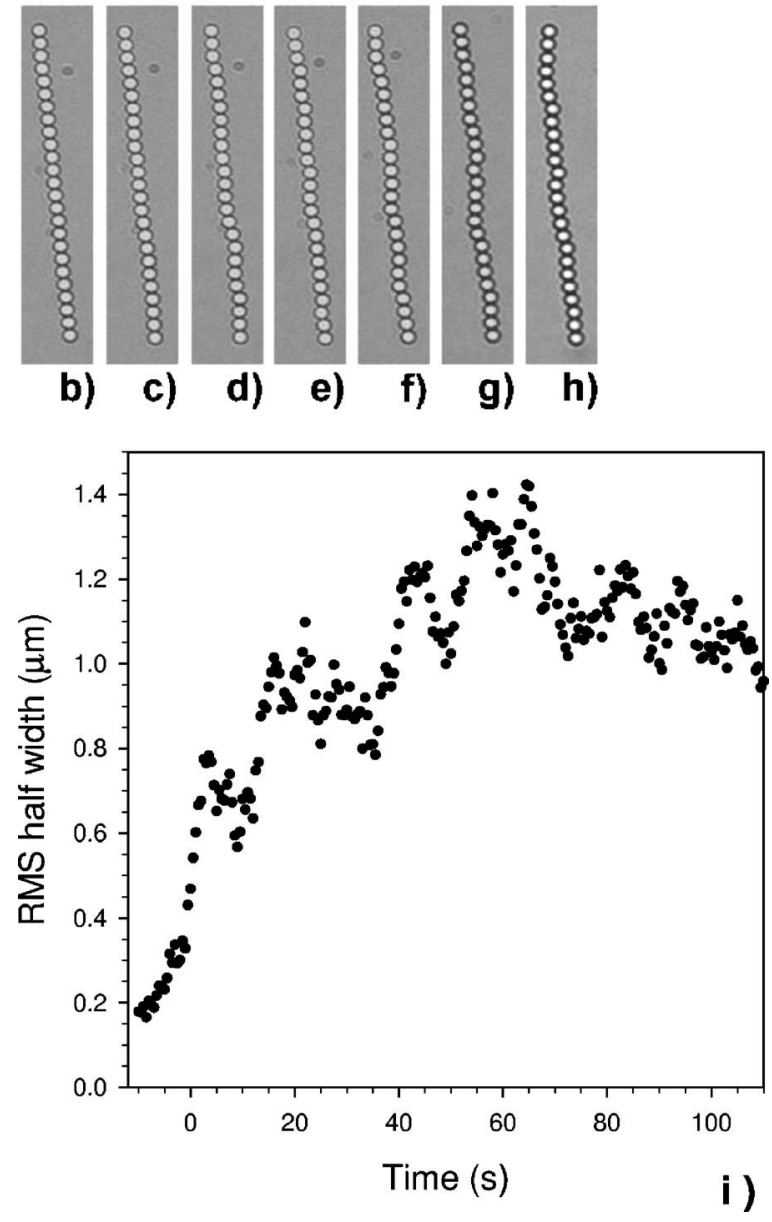

FIG. 2. Dynamic roughening of dipolar chains of magnetic holes of diameter $4 \mu \mathrm{m}$ after a decrease of the external magnetic field from $\lambda=866$ to $\lambda=24$ at $t=0$. (a) Region with low density of chains, (b) the longest chain $10 \mathrm{~s}$ before the field was reduced $(t$ $=-10 \mathrm{~s}$ ), (c) $t=0$, (d) $-(\mathrm{h}) t=3 \mathrm{~s}, 10 \mathrm{~s}, 30 \mathrm{~s}, 60 \mathrm{~s}$, and $150 \mathrm{~s}$, respectively. (i) Time evolution of the root-mean-square half-width of the chain.

\section{ANALYSIS OF LATERAL FLUCTUATIONS}

To obtain the dynamic description of the chain we start from the nearest neighbor interactions and consider departures from the minimum energy configuration of the straight chain.

First we estimate the magnitude of next-nearest-neighbor interactions: From Eq. (1) we see that $U \propto 1 / r^{3}$ and therefore that the ratio of the nearest-neighbor energy to the energy associated with the remaining interactions may be written

$$
\frac{U(a)}{\sum_{n=2}^{\infty} U(n a)} \approx \frac{a U(a)}{\int_{2 a}^{\infty} d r U(r)}=8 .
$$

This we will take as justification to neglect interactions beyond nearest neighbor ones.

To identify the equation of motion for the position $h_{i}$ of particle $i$ in the direction normal to the applied field, we perform a Taylor expansion of the magnetic interaction potential in Eq. (1) around the rigid chain reference state. This gives the normal component of the magnetic force acting on sphere $i$ as

$$
\begin{aligned}
& F_{i}^{M}=k_{\perp}\left(h_{i+1}-2 h_{i}+h_{i-1}\right) \\
& \text { with } k_{\perp}=\pi \mu_{f} \bar{\chi}^{2} H^{2} a / 12,
\end{aligned}
$$

where $a$ is the particle diameter, and $H$ the external applied field. Typically $a \approx 3 \mu \mathrm{m}$, and $H=20 \mathrm{Oe}$, so that $k_{\perp} \approx 1.2$ $\times 10^{-6} \mathrm{~kg} / \mathrm{s}^{2}$.

Since the Reynolds number in this system is very small (using the particle diameter $a=1 \mu \mathrm{m}$ and the thermal particle velocity, $\operatorname{Re}=10^{-4}$ ) the hydrodynamic forces are linear in the particle velocity. Neglecting in a first stage the hydrodynamic interactions between the particles, we write then

$$
F_{i}^{H}=-f_{\perp} h_{i},
$$

where $f_{\perp}=3 \pi \eta a$, with the fluid viscosity $\eta=6 \times 10^{-3}$ Pa s. We will comment further on the influence of the confining cell boundaries on this drag coefficient, and on the influence of the hydrodynamic coupling between the particles in the chain.

Newton's second law for the $i$ th sphere is then

$$
m \ddot{h}\left(x_{i}\right)=F_{M}\left(x_{i}\right)+F_{H}\left(x_{i}\right)+\tilde{F}_{i}(t),
$$

where we have added also the fluctuating force $\widetilde{F}_{i}(t)$, which is due to the molecular nature of the fluid and gives rise to the Brownian motion of the particle.

The correlation time of $\widetilde{F}_{i}(t)$ cannot exceed the viscous damping time $t_{m}=m / f_{\perp}$. This is the time it takes for a particle velocity to decay. An upper bound for $t_{m}$ in our system is given by $t_{m}=a^{2} \rho / 18 \eta \approx 5 \times 10^{-6} \mathrm{~s}$, and above this time scale we may assume

$$
\left\langle\widetilde{F}_{i}(t) \widetilde{F}_{j}(0)\right\rangle=A \delta(t) \delta_{i j},
$$

where $A$ is a constant. In passing we note that since a force over a particle in general causes inertial motion in the fluid, that will decay only over a finite viscous damping time, the equation of motion for $\left\{h_{i}\right\}$ will also in general have a nonMarkovian nature, i.e., the motion of $h_{i}$ will depend not only the instantaneous forces but the entire history of those forces $[32,33]$. However, due to the presence of the confining plates the damping time for any inertial motion is simply $t_{m}$, and above this time it is correct to neglect the non-Markovian corrections to Eq. (7). This argument can also be given a 
more rigorous basis by showing that the non-Markovian interface equations, as given in Ref. [32], actually reduces to Eq. (7) in the $t_{m} \rightarrow 0$ limit.

Having established the Markovian nature of our description without this rigor we now determine $A$. The observation that $\widetilde{F}_{i}$ is independent of $F_{M}$ allows us to determine $A$ from (7) with the $F_{M}$ term removed. Following standard textbook [34] procedures we multiply the remainder of Eq. (7) by $\exp \left[\left(f_{\perp} / m\right) t\right]$ to obtain the explicit solution $\dot{h}\left(x_{i}, t\right)$ $=\int_{-\infty}^{t} d t^{\prime} 1 / m \widetilde{F}_{i}\left(t^{\prime}\right) e^{\left[-\left(f_{\perp} / m\right)\left(t-t^{\prime}\right)\right]}$. If we average the square of this equation, use Eq. (8) and invoke the equipartition principle we obtain $k_{B} T=\left\langle m \dot{h}\left(x_{i}\right)^{2}\right\rangle=A / 2 f_{\perp}$ which gives

$$
A=2 f_{\perp} k_{B} T \text {. }
$$

Combining the above results, and neglecting the inertial terms due to the smallness of $t_{m}$, Eq. (7) can be written

$$
\dot{h}_{i}=\frac{k_{\perp}}{f_{\perp}}\left(h_{i+1}+h_{i-1}-2 h_{i}\right)+\frac{1}{f_{\perp}} \widetilde{F}_{i}(t) .
$$

This is a discrete form of the Edwards-Wilkinson equation, valid when $\lambda \gg 1$ where the quadratic approximation of the potential is valid. For spatial scales above $a$ and times above $t_{m}$, the above reduces to the Edwards-Wilkinson equation [7],

$$
\frac{\partial h}{\partial t}=\frac{k_{\perp} a^{2}}{f_{\perp}} \frac{\partial^{2} h}{\partial x^{2}}+\frac{1}{f_{\perp}} \widetilde{F}_{i}(t),
$$

also known in polymer dynamics as the Rouse model [6].

A chain of particles may be made arbitrarily straight by applying large external magnetic fields. As an idealization of this situation we shall assume an initially straight chain and ask how it roughens when the external fields are reduced, while $\lambda>1$, which ensures that the chain does not melt and the Taylor expansion performed above is accurate enough. As a side result we shall also find the equilibrium width of the chain, that results as a balance between the Brownian forces that tends to buckle the chain and the magnetic forces that tend to straighten it out.

For this purpose we will take the discrete Fourier transform of Eq. (10) as a starting point. The Fourier transforms are defined as

$$
\begin{aligned}
& h_{j}=h\left(x_{j}\right)=\sum_{n=0}^{N-1} e^{i k_{n} x_{j}} h_{n}, \\
& h_{n}=h_{k_{n}}=\frac{1}{N} \sum_{j=0}^{N} h_{j} e^{-i k_{n} x_{j}},
\end{aligned}
$$

where

$$
\begin{gathered}
x_{j}=j a, \quad j=0,1, \ldots, N-1, \\
k_{n}=\frac{2 \pi}{N a} n, \quad n=0, \ldots,(N-1) .
\end{gathered}
$$

The Fourier transform of Eq. (10) may be written

$$
\dot{h}_{n}=-\omega_{n} h_{n}+\frac{1}{f_{\perp}} \widetilde{F}_{n}
$$

where

$$
\omega_{n}=\frac{k_{\perp}}{f_{\perp}} 2\left[1-\cos \left(k_{n} a\right)\right] .
$$

Equation (14) is easily solved to give

$$
h_{n}(t)=\frac{1}{f_{\perp}} \int_{-\infty}^{t} d t^{\prime} e^{-\omega_{n}\left(t-t^{\prime}\right)} \tilde{F}_{n}\left(t^{\prime}\right)
$$

and correspondingly Eq. (8) takes the form-force:

$$
\left\langle\tilde{F}_{n}(t) \tilde{F}_{n^{\prime}}^{*}(0)\right\rangle=\frac{2 f_{\perp} k_{B} T}{N} \delta(t) \delta_{n n^{\prime}} .
$$

The mean square width $W^{2}$ is defined as the average of $\left(h_{i}\right.$ $\left.-1 / N \Sigma_{j} h_{j}\right)^{2}$, which by Parseval's identity may be written

$$
W^{2}=\frac{1}{N} \sum_{i}\left\langle\left(h_{i}-\frac{1}{N} \sum_{j} h_{j}\right)^{2}\right\rangle=\sum_{n>0}\left\langle\left|h_{n}\right|^{2}\right\rangle .
$$

An initially straight particle chain is most conveniently described by setting $\widetilde{F}_{n}(t<0)=0$. The average of the square of $h_{n}(t)$ then takes the form

$$
\begin{aligned}
\left\langle\left|h_{n}(t)\right|^{2}\right\rangle & =\frac{2 k_{B} T}{N f_{\perp}} \int_{0}^{t} d t^{\prime} e^{-2 \omega_{n}\left(t-t^{\prime}\right)} \\
& =\frac{2 k_{B} T}{f_{\perp}} \frac{1-e^{-2 \omega_{n} t}}{2 \omega_{n}}
\end{aligned}
$$

and

$$
W^{2}=\frac{2 k_{B} T}{N f_{\perp}} \sum_{n \geqslant 1} \frac{1-e^{-2 \omega_{n} t}}{2 \omega_{n}} .
$$

When $t \ll 1 / \omega_{\max }$ we have $\left[1-\exp \left(-2 \omega_{n} t\right)\right] /\left(\omega_{n} t\right) \approx 2$ and

$$
W^{2} \approx \frac{2 k_{B} T}{f_{\perp}} t .
$$

This result arises only because of the existence of a shortest wavelength $a$ in the system. In the continuum limit $a \rightarrow 0$, $\omega_{\max } \rightarrow \infty$, and the $t \ll 1 / \omega_{\max }$ regime does not exist. Equation (22) describes the superposition of $N$ independent random walks in the $h$ direction, and therefore the $t^{1 / 2}$ dependence of $W$ is as expected.

When $1 / \omega_{\max } \ll t \ll 1 / \omega_{\min }$, then $\omega_{k} t \gg 1$ for large $k$ and $\omega_{k} t \ll 1$ for small $k$. Since $\left[1-\exp \left(-2 \omega_{n} t\right)\right] /\left(\omega_{n} t\right)$ is of order 1 for the smallest $\omega_{k}$ and $\left[1-\exp \left(-2 \omega_{n} t\right)\right] /\left(\omega_{n} t\right) \ll 1$ for large $\omega_{k}$ we see that the sum over wave numbers is dominated by the small $\omega_{k}$. We can then write $\omega_{k} \approx(k a)^{2} / \tau$ where $\tau$ $=f_{\perp} / k_{\perp} \approx 0.2(H / 20 \mathrm{Oe})^{2} \mathrm{~s}$. By noting that $\omega_{N-n}=\omega_{n}$, the mean square width $W^{2}$ may be cast in the Family-Vicsek scaling form [35]

$$
W^{2}=L F\left(\frac{t}{L^{2}}\right)
$$

where 


$$
F(x)=\frac{k_{B} T}{2 \pi^{2} k_{\perp} a} \sum_{n=1}^{N / 2} \frac{1-e^{-8 k_{\perp} a^{2} \pi^{2} n^{2} x / f_{\perp}}}{n^{2}} .
$$

This equation has the following physical interpretation. Every mode $k_{n}$ grows to its equilibrium value on a time scale $1 / \omega_{n}$. At that point there is an equilibrium between the effects of the thermal force $\widetilde{F}_{i}$ and the restoring force $F_{M_{i}}$. Since at small times $W$ must be independent of $L$ and at large times, when $W$ has reached its asymptotic value, it must be independent of $t$, we have that

$$
F(x) \sim\left\{\begin{array}{cc}
x^{1 / 2} & \text { for small } x, \\
\text { const } & \text { for large } x .
\end{array}\right.
$$

From this it follows that

$$
W \sim \begin{cases}t^{1 / 4} & \text { for small } t \\ L^{1 / 2} & \text { for large } t .\end{cases}
$$

To get the prefactors however, we must evaluate the sums. In the $1 / \omega_{\max } \ll t \ll 1 / \omega_{\min }$ regime we can replace the sum by an integral to a good approximation. This yields

$$
W^{2}(t)=\frac{2 k_{B} T t}{N f_{\perp} \Delta k} \int_{2 \pi / N a}^{\pi / a} d k \frac{1-e^{-2 \omega_{k} t}}{\omega_{k} t},
$$

where $\Delta k=2 \pi /(N a)$. Using the small $k$ expansion for $\omega_{k}$ and a simple substitution we obtain

$$
W^{2}(t) \approx \frac{k_{B} T \tau}{f_{\perp} \pi a} \int_{0}^{\infty} d k \frac{1-e^{-k^{2}\left(2 a^{2} t / \tau\right)}}{k^{2}}=\frac{k_{B} T}{\sqrt{k_{\perp} f_{\perp}}} I t^{1 / 2},
$$

where

$$
I=\frac{\sqrt{2}}{\pi} \int_{0}^{\infty} d x \frac{1-e^{-x^{2}}}{x^{2}}=\sqrt{\frac{2}{\pi}} .
$$

When $t \gg 1 / \omega_{\min }, e^{-2 \omega_{n} t} \approx 0$ for all $n$, and

$$
W^{2}=\frac{2 k_{B} T}{N f_{\perp}} \sum_{n=1}^{N / 2} \frac{1}{\omega_{n}} \approx \frac{k_{B} T}{2 \pi^{2} k_{\perp}} \frac{L}{a} \sum_{n=1}^{\infty} \frac{1}{n^{2}} .
$$

Using

$$
J=\frac{1}{2 \pi^{2}} \sum_{n=1}^{\infty} \frac{1}{n^{2}}=\frac{1}{12}
$$

we may summarize the above as

$$
W^{2}=k_{B} T\left\{\begin{array}{cc}
\frac{2}{f_{\perp}} t & \text { when } t \ll \frac{1}{\omega_{\max }}, \\
\sqrt{\frac{2}{\pi k_{\perp} f_{\perp}}} t^{1 / 2} & \text { when } \frac{1}{\omega_{\max }} \ll t \ll \frac{1}{\omega_{\min }}, \\
\frac{1}{12 k_{\perp}} \frac{L}{a} & \text { when } t \gg \frac{1}{\omega_{\min }} .
\end{array}\right.
$$

Noting finally from Eqs. (13) and (15) that $1 / \omega_{\max }=\tau / 4$ and $1 / \omega_{\min }=N^{2} \tau / 4 \pi^{2}$, those separation times can be evaluated as $1 / \omega_{\max }=0.06(H / 20 \mathrm{Oe})^{2} \mathrm{~s}$ and $1 / \omega_{\min }$ $=N^{2} \cdot 0.06(H / 20 \mathrm{Oe})^{2} \mathrm{~s}$.

\section{BROWNIAN DYNAMIC SIMULATIONS}

The dynamic roughening of such dipolar chain can also be studied numerically by performing Brownian dynamics simulations. In this model the interaction forces, which act on each particle of the chain, is composed of the dipoledipole interaction of Eq. (1), a repulsive contact force, and a thermal, fluctuating force from the surrounding fluid. Such a numerical study allows us to study the effects neglected in the analytical derivation above, namely, (1) the finite range of the dipolar interactions beyond nearest neighbor ones. (2) The influence of the nonlinearities in the potential for large departures from a straight chain. (3) The fact that particles are not constantly in contact but have somewhat fluctuating spacings.

Our hydrodynamic regime is strongly damped, which implies that the acceleration term $m_{i} \ddot{\mathbf{r}}$ is negligible compared to the viscous friction term $\mathbf{F}_{\mathbf{v}}=-\mathbf{f}_{\perp} \dot{\mathbf{r}}_{\mathbf{i}}$. The time evolution of each particle in the chain is then given by the force-balance equation

$$
f_{\perp} \dot{\mathbf{r}}_{i}=-\left(\sum_{j} \mathbf{F}_{m}\left(\mathbf{r}_{j}-\mathbf{r}_{i}\right)\right)+\mathbf{F}_{c}\left(\mathbf{r}_{j}-\mathbf{r}_{i}\right)+\widetilde{\mathbf{F}}_{i}(t),
$$

where the magnetic interaction force is derived from the pair potential $U$ in Eq. (1), i.e., $\mathbf{F}_{\mathbf{m}}(\mathbf{r})=-\nabla \mathbf{U}$.

The contact forces are modeled as

$$
\mathbf{F}_{c}(\mathbf{r})=f_{0} \Theta(a-r) \hat{\mathbf{r}} \exp \left(\frac{r-a}{l}-\frac{l}{r-a}\right),
$$

where $\Theta$ is a Heavyside function, $r-a$ the overlap distance between neighboring particles, $l$ a characteristic distance of overlap, $\hat{\mathbf{r}}$ the radial unit vector, and $f_{0}$ the characteristic force magnitude arising from the elastic properties of the particle material. The shape of this interaction is chosen so that this force and its derivative of any order are continuous-although similar results are obtained with the simpler form $\mathbf{F}_{c}(\mathbf{r})=f_{0} \Theta(a-r) \hat{\mathbf{r}} \exp [(r-a) /(l)]$. In the model, we adopt arbitrarily $l=0.01 a$, and $f_{0}=U(r=a, \theta$ $=0) / a$. By testing several values of these parameters, we have checked that these particular values are irrelevant for the roughening dynamic, as long as $l / a \ll 1$, which ensures that the interactions approximate those of rigid particles.

The hydrodynamic interactions between the plates and the particles cannot be neglected. They are taken into account in the following way.

Since the magnetic interactions between the particles and the nonmagnetic boundaries keep the particles in the middle between the plates, the hydrodynamic coupling between the particles and the plates amount to increasing the friction coefficient by a factor

$$
c=2 /(1-9 a / 16 d)-1=1.40(1.70)
$$

for $\mathrm{a}=3 \mu \mathrm{m}(4 \mu \mathrm{m})$ [36]. Consequently, the viscous friction term is modeled as $\mathbf{F}_{v}=-f_{\perp} \dot{\mathbf{r}}_{i}$, with $f_{\perp}=3 c \pi \eta a$. Eventually, 

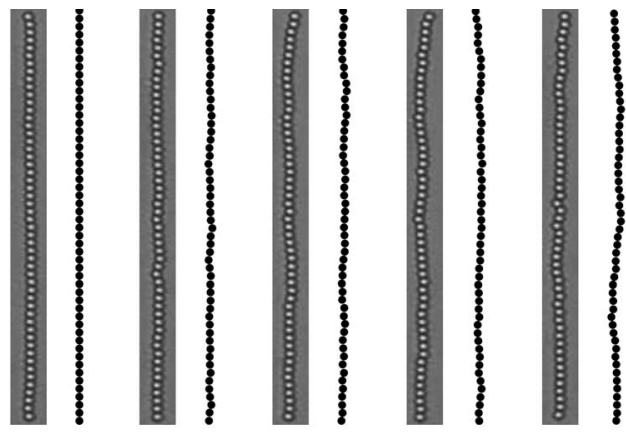
(a) (b)
(c) (d)
(e) (f)
(g) (h)
(i) (j)
$0 \mathrm{~s} \quad 0 \mathrm{~s}$
$10 \mathrm{~s} 10 \mathrm{~s}$
40s 40s
90s 90s
200s 200s

FIG. 3. Typical dynamic roughening of dipolar chains of 52 magnetic holes of diameter $4 \mu \mathrm{m}$ after a decrease of the external magnetic field from $\lambda=866$ to $\lambda=24$ at initial time, experiments (gray) and simulations (black and white).

the hydrodynamic interactions between the particles are neglected, as will be justified further in Sec. V B.

The magnitude of the fluctuating force in the Langevin equation, Eq. (33), is set up by the fluctuation-dissipation theorem, as derived in Eqs. (9), i.e.,

$$
\left\langle\widetilde{F}_{i}(t) \widetilde{F}_{j}(0)\right\rangle=2 f_{\perp} k_{B} T \delta(t) \delta_{i j} .
$$

The Langevin equation (33) is directly integrated over time steps $\Delta t$ as

$$
\begin{aligned}
f_{\perp}\left[\mathbf{r}_{i}(t+\Delta t)\right. & \left.-\mathbf{r}_{i}(t)\right] \\
= & -\left(\sum_{j} \mathbf{F}_{m}\left[\mathbf{r}_{j}(t)-\mathbf{r}_{i}(t)\right]\right) \Delta t \\
& +\mathbf{F}_{c}\left[\mathbf{r}_{j}(t)-\mathbf{r}_{i}(t)\right] \Delta t+\Delta \tilde{\mathbf{F}}_{i}(t),
\end{aligned}
$$

where according to the central limit theorem and Eq. (36), the integrated random variables $\Delta \widetilde{\mathbf{F}}_{i}=\int_{t}^{t+\Delta t} \widetilde{\mathbf{F}}_{i}\left(t^{\prime}\right) d t^{\prime}$ are uncorrelated Gaussian variable of zero mean and of variance given by

$$
\left\langle\tilde{F F}_{i}(t) \Delta \tilde{F F}_{j}\left(t^{\prime}\right)\right\rangle=2 f_{\perp} k_{B} T \Delta t \delta_{t t^{\prime}} \delta_{i j} .
$$

The time step $\Delta t$ is chosen so that the average spatial steps do not exceed 0.001 diameters. The simulations are run from an initial state where all particles are in contact and aligned.

As in the experiments, under the effect of thermal noise, the chains roughen until they reach a characteristic fluctuation size. The equilibrium size of these fluctuations depend on the applied field. Five snapshots of a typical simulation are shown in Fig. 3, together with an experiment carried out with similar field parameters. In the experiment, the field was reduced from a strong value corresponding to $\lambda=866$, to a lower one corresponding to $\lambda=24$. In the simulation, the initial straight state corresponding to $\lambda \gg 1$ is let to fluctuate according to the Brownian dynamic scheme carried out with restoring magnetic forces at $\lambda=24$. The visual agreement between simulations and experiments seems satisfying, as shown in Fig. 3.

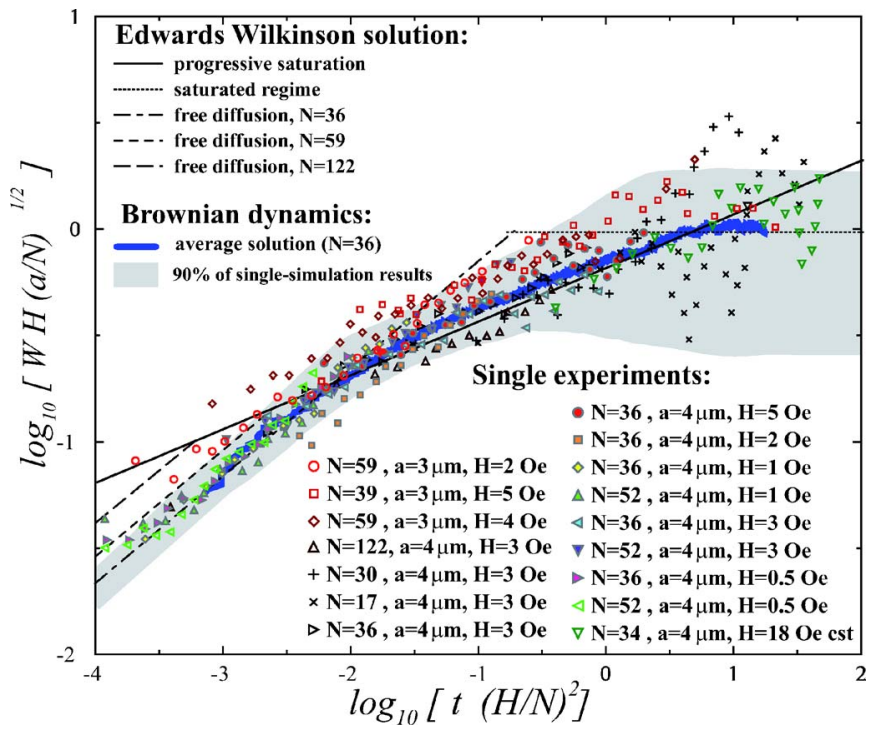

FIG. 4. (Color online) Collapse of the scaled rms of the displacements, as a function of the scaled time: experimental data compared to analytical theory, with average behavior and characteristic variance evaluated from simulations. The units of $H, t$, and $W$ are, respectively, Oersteds, seconds and $\mu m$.

\section{COMPARISON BETWEEN THEORETICAL MODEL, SIMULATIONS AND EXPERIMENTS}

\section{A. Evolution of the chain width}

To characterize the roughening dynamic of the dipolar chains, we extract the root-mean-square width of the lateral coordinates as function of time $t$ after a decrease of the external field from a strong value. Since this system is excited by thermal noise, this width differs between realizations. We record this width as a function of time for each single experiment or numerical realization of Brownian dynamics simulation,

$$
W_{s r}(t)=\frac{1}{N} \sum_{j} h_{j}^{2}
$$

The average over realizations of this rms width should correspond to the thermodynamic average $W(t)=\left\langle W_{s r}\right\rangle$ derived in Sec. III. In order to check the scaling law derived in Eq. (32), for the width as function of time, we plot the scaled width $W H \sqrt{a / N}$ as function of the scaled time, $t c(H / N)^{2}$, in Fig. 4. Each symbol represents a different experiment, with a different applied field, particle number or particle diameter, as specified in the figure.

The black continuous lines correspond to the predicted thermodynamic average, evaluated from Eq. (32) with $f_{\perp}$ $=3 c \pi \eta a$ with $c$ given by Eq. (35). The dashed lines correspond to the initial freely diffusive regime for chains of length 36, 59, and 122. The data collapse of the experimental data in these reduced coordinates is consistent with the analytical model, but the scatter around the thermodynamic average is non-negligible for each of these single experiments, as is expected when no averaging is performed.

To characterize further this scatter around the average, we have performed the following analysis on the Brownian 


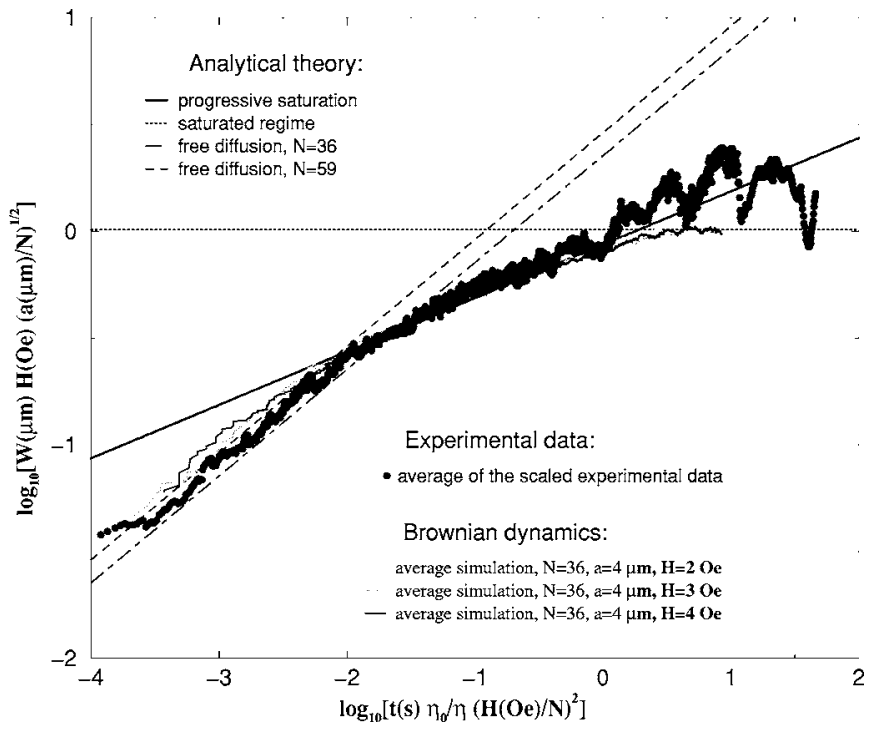

FIG. 5. Scaling of the random mean square width of the transverse displacements: theory, experiments and Brownian dynamics result.

dynamics simulation: From 1000 different runs on 36 particles long chains, we have evaluated the average width over these realizations at a given time, $\left\langle W_{n}(t)\right\rangle$. This is plotted in Figs. 4 and 5 for fields of magnitude $H=2,3$, and 4 Oe, and particles of $3 \mu \mathrm{m}$. These three average sets indeed collapse in reduce coordinates, and correspond reasonably well to the analytical estimates shown as continuous lines.

Each of these simulations nonetheless exhibits a dynamic width $W_{n}(t)$ departing significantly from the thermodynamic average. At each time, we have evaluated the standard deviation of the width from 1000 simulations, $\sigma(t)$ $=\sqrt{\left\langle W_{n}^{2}(t)-\left\langle W_{n}(t)^{2}\right\rangle\right\rangle}$. In reduced coordinates, $\sigma / W$ at a given scaled time is found to be lowly sensitive to $H$. The distribution of the width at a given time is essentially Gaussian, and at each time $t, \sqrt{2 / \pi} \operatorname{erfc}(2 / \sqrt{2})=95 \%$ of the widths $W_{n}(t)$ of single numerical realizations are within $2 \sigma(t)$ of the thermodynamic average $\langle W(t)\rangle$.

In Fig. 4, we have represented in gray shading the zone corresponding to this probable interval of $95 \%$ of the numerical realizations, $[\langle W(t)\rangle-2 \sigma(t) ;\langle W(t)\rangle+2 \sigma(t)]$. Indeed, most experimental widths evaluated for single experiments are in this range, i.e., the experimental deviation from the average for a given realization is comparable to the one observed in the Brownian dynamics simulations. So, the data collapse of the experimental reduced width as function of the reduced time seems satisfactory.

To extract the average behavior from the experiments, we performed a running average of the scaled width over all 16 experiments, with a logarithmic binning procedure so as to have 40 points per time decade, i.e., averages over time windows $\left[t_{i}, t_{i+1}\right]$ with $t_{i+1}=\alpha t_{i}$, with $\alpha=10^{1 / 40}=1.05$. The resulting average over experiments, represented in black circular symbols in Fig. 5, coincides reasonably well with the Brownian dynamics and analytical results. The larger discrepancies are observed for the largest reduced times, where only a few experiments have been carried out (at large times in the larg- est fields), and can be interpreted once again as an insufficient number of independent experimental realizations. Moreover, this happens in the region where the variance of the width of a single realization is the largest, due to the fact that the largest wavelengths modes are developed, and that the number of such wavelengths in the system is limited, so that no spatial average smoothens the effect of the fluctuations of these large wavelengths.

The tunability of the characteristic time as function of the applied field in our system allows to effectively sample experimentally more than five decades of reduced times. This allows to distinguish experimentally the three regimes predicted, $W \sim t^{1 / 2}, W \sim t^{1 / 4}$ and saturation width, each of them being observed over a couple of decades.

\section{B. Irrelevance of hydrodynamic interactions between particles in a confined system}

The reason for neglecting the hydrodynamic interactions in this setup is the presence of the plates confining the system dampen such hydrodynamic interactions. Indeed, the corrective force on particle $i$ due to the hydrodynamic interaction with the particle $j$, moving at velocity $v_{j}$ with respect to the fluid at infinity, can be estimated as $f_{\perp} u_{i j}$, where $u_{i j}$ is the characteristic fluid velocity at the center of particle $i$, that the particle $j$ moving at $v_{j}$ would create in a fluid medium with no other particles [6]. At scales larger than the plate separation, the pressure field between two parallel plates is Laplacian, i.e., $\nabla^{2} P=0$. Indeed, the average fluid velocity between two parallel plates $u$ can be obtained via Darcy's law, $u \propto-\nabla P[37]$, since the ferrofluid is incompressible, $\nabla \cdot u=0$. A moving particle generates a dipolar pressure source perturbation, decaying as $1 / r$ and a fluid velocity perturbation decaying as $u \sim-\nabla P \sim 1 / r^{2}$. Thus, the flow perturbation generated by the thermal motion of all particles further than a distance $l$ behaves as $\sum_{j>l / a} v_{j} a^{2} /(j a)^{2}$. An upper bound for this corrective velocity contribution can be set as $v_{T} a / l$ where $v_{T} \sim \sqrt{k_{B} T / m}$ is the characteristic thermal velocity magnitude. For large enough $l$, this correction can be made arbitrarily small, and consequently, the hydrodynamic interactions between particles remain of finite range. Taking into account the hydrodynamic interactions between a few neighboring particles could possibly induce a correction as a prefactor for the rms width of the chain, which, since it only corrects local effects, would not change the exponents.

Indeed, we have observed that the experiments agree well with the theoretically predicted scaling laws $W \sim t^{\alpha}$. Furthermore, even the prefactor $W(t) / t^{\alpha}$ is captured by theory and simulations that neglect the hydrodynamic interactions between particles. This shows a posteriori that these interactions are indeed irrelevant for our confined system.

On the other hand, for three-dimensional systems where no confining plates dampen such hydrodynamic pertubations, a particle moving at velocity $v$ induces a flow perturbation decaying as $v \cdot a / r$, as given by the Oseen tensor in an unbounded medium [6]. The sum of these perturbations for particles beyond a certain distance $l, \Sigma_{j>l / a} v_{j} a /(j a)$, depends on the precise series of velocities $v_{j}$, and cannot be trivially bounded. Consequently, hydrodynamic interactions for dipo- 
lar chains in unconfined fluids are in general relevant, and their presence leads to a different anomalous diffusion exponent, $W \sim t^{1 / 3}$ [6]. This can be described by the Zimm model, and experiments carried out on magnetorheological fluids in three-dimensional geometries indeed report $W \sim t^{0.35 \pm 0.05}$ [9-12].

\section{EQUILIBRIUM FLUCTUATIONS}

So far, we have studied how an initially straight chain roughens dynamically when the external field is suddenly lowered to a finite and constant value. This out-ofequilibrium problem corresponds to following the dynamic roughening of our dipolar chain under the effect of a thermal bath turned on initially.

Once the chain reaches equilibrium it is a different problem to characterize the growth away from an arbitrary state of the equilibrium distribution. This problem is hence an equilibrium one, and we will now characterize the spatial and temporal scaling of the autocorrelation function.

The difference between these equilibrium fluctuations and the out-of-equilibrium dynamic roughening problem lies only in the knowledge of the initial state: The roughening process is nonequilibrium because it starts from a nonequilibrium distribution, that is, a distribution of (flat) states that are only a subset of all the equilibrium states. For the equilibrium process we consider the departure from an arbitrary rough state $h\left(x_{j}, t_{0}\right)$, i.e.,

$$
\Delta h\left(x_{j}, t, t_{0}\right)=h\left(x_{j}, t+t_{0}\right)-h\left(x_{j}, t_{0}\right) .
$$

As in the out-of-equilibrium problem, this quantity starts at 0 at $t=0$. We can characterize the rms width of this departure, as

$$
\begin{aligned}
\Delta W^{2}\left(t, t_{0}\right) & =\frac{1}{N} \sum_{j=1}^{N}\left(\Delta h_{j}-\frac{1}{N} \sum_{l} \Delta h_{l}\right)^{2} \\
& =\sum_{n=1}^{N-1}\left\langle\left|\widetilde{\Delta} h_{n}\left(t, t_{0}\right)\right|^{2}\right\rangle,
\end{aligned}
$$

where we used Parseval theorem.

To evaluate the contribution of each Fourier mode, we integrate once again the Langevin equation, Eq. (14), to get

$$
\begin{aligned}
\Delta h_{n}\left(t, t_{0}\right) & =h_{n}(t)-h_{n}\left(t_{0}\right) \\
& =h_{n}\left(t_{0}\right)\left(e^{-\omega_{n} t}-1\right)+\frac{1}{f_{\perp}} \int_{t_{0}}^{t_{0}+t} d t^{\prime} e^{-\omega_{n}\left(t_{0}+t-t^{\prime}\right)} \widetilde{F}_{n}\left(t^{\prime}\right) .
\end{aligned}
$$

Using the expression for the variance of the fluctuating forces, $\left\langle\widetilde{F}_{n}(t) \widetilde{F}_{n^{\prime}}^{*}(0)\right\rangle=2 f_{\perp} k_{B} T / N \delta(t) \delta_{n n^{\prime}}$, we can integrate this to obtain

$$
\begin{aligned}
\left\langle\Delta h_{n}\left(t, t_{0}\right)^{2}\right\rangle= & \left\langle h_{n}\left(t_{0}\right)^{2}\right\rangle\left(e^{-2 \omega_{n} t}-2 e^{-\omega_{n} t}+1\right) \\
& +2 k_{B} T \int_{t_{0}}^{t_{0}+t} e^{-\omega_{n}\left(2 t_{0}+2 t-2 t^{\prime}\right)} d t^{\prime}
\end{aligned}
$$

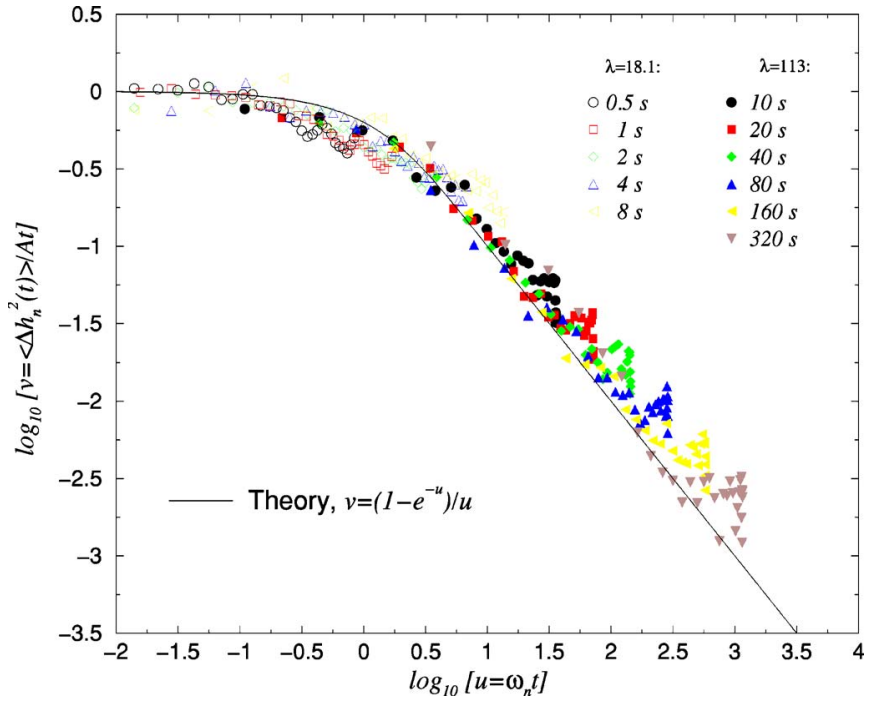

FIG. 6. (Color online) Power spectrum of the fluctuations: scaling of the autocorrelation function in time and space.

$$
\begin{aligned}
= & \left\langle h_{n}\left(t_{0}\right)^{2}\right\rangle\left(e^{-2 \omega_{n} t}-2 e^{-\omega_{n} t}+1\right) \\
& +k_{B} T \frac{\left(1-e^{-2 \omega_{n} t}\right)}{\omega_{n}} .
\end{aligned}
$$

The equilibrium width of mode $n$ is given by Eq. (19) at large times, i.e., $\left\langle h_{n}\left(t_{0}\right)^{2}\right\rangle=k_{B} T / \omega_{n}$. Then the above equation simplifies to

$$
\left\langle\Delta h_{n}\left(t, t_{0}\right)^{2}\right\rangle=2 k_{B} T\left(1-e^{-\omega_{n} t}\right) / \omega_{n} .
$$

The dynamics of this fluctuating mode is independent of the initial time $t_{0}$, reflecting the fact that equilibrium averages are time translational invariant. We note that the time dependence of $\left\langle\Delta h_{n}\left(t, t_{0}\right)^{2}\right\rangle$ is closely related to the time dependence of the roughening states in Eq. (19): The behavior is in fact identical, only with a doubled prefactor, and a doubled characteristic time. This is linked to the fact that the chain may on the average displace further from an arbitrary state than a straight one.

The above scaling law for the dynamic departure from a given equilibrium configuration was directly checked in two series of experiments, Eq. (51) predicts that

$$
\left\langle\left[\Delta h_{n}\left(t, t_{0}\right)\right]^{2}\right\rangle / A t=f(u),
$$

where $u=\omega_{n} t, f(u)=\left(1-e^{-u}\right) / u$ and $A=2 k_{B} T$.

The two experiments were performed at fields such that $\lambda=18$ and $\lambda=113$, and we recorded 20 minutes of chain motion, for which we performed the spatial Fourier transform of $\Delta h\left(x, t, t_{0}\right)$. A Hamming window was used to avoid frequency leakage due to the finite chain sizes [38].

We subsequently evaluated the power spectrum $\Delta h_{n}\left(t, t_{0}\right)^{2}$ which was averaged over $t_{0}$ with a fixed time lapse $t$. The result is plotted in the scaled form $\left\langle\left[\Delta h_{n}\left(t, t_{0}\right)^{2}\right]\right\rangle_{t_{0}} / A t$ as a function of $u=\omega_{n} t$ in Fig. 6. Note that each curve actually corresponds to fixed $t$ and variable $n$. The fact that $t$ varies by more than two orders of magnitude thus gives a five order of magnitude variation in $u$. 
Both experiments at all probed times show a fluctuation spectrum consistent with the theoretical prediction of Eq. (52), which is shown as a continuous line. The initial flat plateau corresponds first to the normal diffusion regime where $f(u) \sim 1$, and then the progressive saturation of the modes is seen as the straight line of slope -1 on the righthand side, i.e., a power-law decay with $f(u) \sim 1 / u$.

Note that the knowledge of the spatial power spectrum $\left\langle\widetilde{\Delta} h_{n}^{2}\right\rangle$ and the equilibrium average $\left\langle h_{n}^{2}\right\rangle$ given in Eq. (19) allows us also to evaluate the two-point correlation function in space and time. This function is defined as

$$
G(x, t)=\langle h(0,0) h(x, t)\rangle,
$$

which has the spatial Fourier transform

$$
G_{n}(t)=\left\langle h_{n}(0) h_{n}(t)\right\rangle .
$$

By definition of $\Delta h_{n}(t)$ with $t_{0}=0$ we can write

$$
\left\langle\Delta h_{n}(t)^{2}\right\rangle=\left\langle h_{n}(t)^{2}\right\rangle-2\left\langle h_{n}(t) h_{n}(0)\right\rangle+\left\langle h_{n}(0)^{2}\right\rangle,
$$

where the first and last right-hand-side terms are the same equilibrium averages.

Solving for $G_{n}(t)$ above we immediately get

$$
\begin{aligned}
G_{n}(t) & =\left\langle h_{n}^{2}\right\rangle-\frac{\left\langle\Delta h_{n}(t)^{2}\right\rangle}{2}=k_{B} T\left(\frac{1}{\omega_{n} t}-\frac{1-e^{-2 \omega_{n} t}}{\omega_{n}}\right) \\
& =\frac{e^{-2 \omega_{n} t}}{\omega_{n}} k_{B} T,
\end{aligned}
$$

where we have used Eq. (51) with $t_{0}=0$ to substitute for $\left\langle\Delta h_{n}(t)^{2}\right\rangle$.

\section{THE MEMORY OF THE BROWNIAN WORM}

In this section, we will show that the $W \sim t^{1 / 4}$ regime can also be understood in terms of anomalous diffusion in a system with memory. We will show how the Markovian description for the entire chain becomes a non-Markovian description when it is formulated for a single particle in the chain.

For such a particle linked to a background constituted of both the solvent and the rest of the chain, the motion is governed by an exterior with long-time memory in the fluctuating force as well as the response function.

The single particle description takes the form of a generalized Langevin equation of the Mori-Lee form [8]:

$$
m \ddot{h_{0}}(t)=-m \int_{-\infty}^{t} d t_{1} \Gamma\left(t-t_{1}\right) \dot{h}_{0}\left(t_{1}\right)+F(t),
$$

with $\Gamma(t)$ a linear response function, and $F(t)$ a fluctuating function satisfying the fluctuation dissipation theorem,

$$
\langle F(t) F(0)\rangle=m k_{B} T \Gamma(t) .
$$

From the theory of anomalous diffusion in such systems with memory, the anomalous diffusion exponent is related to the asymptotic behavior of the linear response function [8]. When the Laplace transform of the response function behaves as $\hat{\Gamma}(\omega) \sim \omega^{-\alpha}$ when $\omega \rightarrow 0$, this theory predicts an anomalous diffusion $W^{2} \sim t^{1-\alpha}$. We will show that this system indeed follows this theoretical prediction, i.e., that it can be described by an Eq. (57) with the asymptotic behavior $\hat{\Gamma}(\omega) \sim \omega^{-1 / 2}$. In other words, this alternative description also produces the prediction $W \sim t^{1 / 4}$, in agreement with the direct treatment above.

\section{A. Generalized Langevin equation for a particle in a Brownian worm}

We consider a set of $N$ particles in an immobile solvent. The inertial terms are neglected for every particle. However, for technical purposes we shall take the particle in focus to have a mass initially. This is done to exhibit a known structure of the Langevin equation: eventually, the mass of this particle will also be send to zero.

Thus, we write for every particle $j$,

$$
\delta_{j 0} m \ddot{h_{j}}=-\kappa \dot{h}_{j}+\alpha\left(h_{j+1}-2 h_{j}+h_{j-1}\right)+\zeta_{j},
$$

where $\kappa=3 \pi \eta a$ and $\alpha$ is the curvature of the magnetic energy with respect to lateral bending, and $\zeta_{j}$ is the uncorrelated Brownian force with a magnitude which is given by dissipation fluctuation theorem

$$
\left\langle\zeta_{i}(0) \zeta_{j}(t)\right\rangle=2 \kappa k_{B} T \delta_{i j} \delta(t) .
$$

We Fourier transform this in space

$$
f_{n}^{\prime}=\sum_{j=1}^{N} f_{j} e^{-2 \pi j n / N}, \quad f_{j}=\frac{1}{N} \sum_{n=0}^{N-1} f_{n}^{\prime} e^{2 \pi j n / N} .
$$

From here on primes refer to Fourier modes in space, and tildes to Fourier modes in time to simplify notation. We then obtain

$$
-m \ddot{h_{0}}=\kappa\left(\dot{h}_{n}^{\prime}+\omega_{n} h_{n}^{\prime}\right)-\zeta_{n}^{\prime}=\kappa e^{-\omega_{n} t} \frac{d}{d t}\left[h_{n}^{\prime}(t) e^{\omega_{n} t}\right]-\zeta_{n}^{\prime},
$$

where

$$
\omega_{n}=\frac{2 \alpha}{\kappa}\left[1-\cos \left(\frac{2 \pi n}{N}\right)\right]
$$

i.e., when

$$
n \ll N, \quad \omega_{n} \simeq \omega_{1} n^{2},
$$

where

$$
\omega_{1}=\frac{\alpha}{\kappa} \frac{(2 \pi)^{2}}{N^{2}} .
$$

Equation (62) is integrated as

$$
-h_{n}^{\prime}(t)=\int_{-\infty}^{\infty} d t_{1}\left[m \ddot{h}_{0}\left(t_{1}\right)-\zeta_{n}^{\prime}\left(t_{1}\right)\right] \frac{\Theta\left(t-t_{1}\right) e^{-\omega_{n}\left(t-t_{1}\right)}}{\kappa},
$$

where $\Theta$ is the Heaviside function. Performing an inverse space Fourier transform, we get 


$$
\begin{aligned}
-h_{0}(t)= & \int_{-\infty}^{\infty} d t_{1} m \ddot{h}_{0}\left(t_{1}\right) \frac{1}{N} \sum_{n} \frac{\Theta\left(t-t_{1}\right) e^{-\omega_{n}\left(t-t_{1}\right)}}{\kappa} \\
& -\int_{-\infty}^{\infty} d t_{1} \frac{1}{N} \sum_{n} \zeta_{n}^{\prime}\left(t_{1}\right) \frac{\Theta\left(t-t_{1}\right) e^{-\omega_{n}\left(t-t_{1}\right)}}{\kappa} .
\end{aligned}
$$

Taking the derivative with respect to time, we obtain

$$
\begin{aligned}
\int_{-\infty}^{\infty} d t_{1} m \ddot{h}_{0}\left(t_{1}\right) \sum_{n} \frac{\delta\left(t-t_{1}\right)-\Theta\left(t-t_{1}\right) \omega_{n} e^{-\omega_{n}\left(t-t_{1}\right)}}{\kappa N} \\
=-\dot{h}_{0}(t)+\int_{-\infty}^{\infty} d t_{1} \sum_{n} \zeta_{n}^{\prime}\left(t_{1}\right) \frac{\delta\left(t-t_{1}\right)-\Theta\left(t-t_{1}\right) \omega_{n} e^{-\omega_{n}\left(t-t_{1}\right)}}{\kappa N} .
\end{aligned}
$$

Using $*$ as a convolution operator we may write the above as

$$
m \ddot{h_{0}} * G=-\dot{h}_{0}+\sum_{n} \zeta_{n} * G_{n},
$$

where

$$
\begin{gathered}
G_{n}(t)=\frac{\delta(t)-\Theta(t) \omega_{n} e^{-\omega_{n} t}}{\kappa N}, \\
G(t)=\sum_{n} G_{n}(t) .
\end{gathered}
$$

We now perform a time Fourier transform of the above. This transform is defined as

$$
\tilde{f}(\omega)=\int_{-\infty}^{\infty} f(t) e^{-i \omega t} d t, \quad f(t)=\frac{1}{2 \pi} \int_{-\infty}^{\infty} \tilde{f}(\omega) e^{i \omega t} d \omega .
$$

The convolution theorem then yields

$$
m \tilde{\ddot{h}}_{0}(\omega)=-m \widetilde{\Gamma}(\omega) \tilde{\dot{h}}_{0}(\omega)+\widetilde{F}(\omega),
$$

where

$$
\begin{gathered}
m \widetilde{\Gamma}(\omega)=1 / \widetilde{G}(\omega), \\
\widetilde{F}(\omega)=\sum_{n} \widetilde{\zeta}_{n}^{\prime}(\omega) \widetilde{G}_{n}(\omega) / d e G(\omega) .
\end{gathered}
$$

An inverse Fourier transform, then leads to

$$
m \ddot{h}_{0}(t)=-m \int_{-\infty}^{t} d t_{1} \Gamma\left(t-t_{1}\right) \dot{h}_{0}\left(t_{1}\right)+F(t)
$$

which is exactly the Mori-Lee generalized Langevin equation [8]. To replace the $+\infty$ upper bound of the integral by $t$, we have used that the response function is causal, i.e., that $\Gamma(t)=0$ when $t<0$ : This is shown explicitly in Appendix A. The fact that the generalized fluctuation dissipation theorem is satisfied, i.e., that

$$
\langle F(t) F(0)\rangle=m k_{B} T \Gamma(t)
$$

is verified in Appendix B.

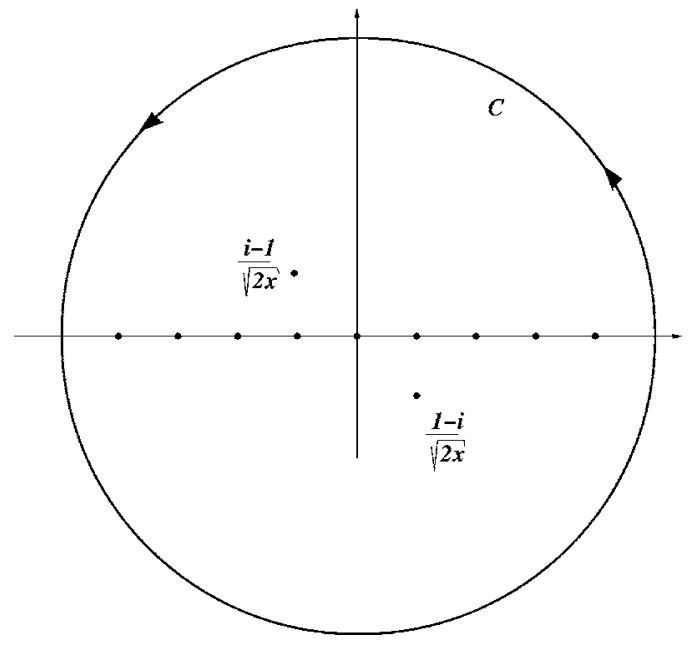

FIG. 7. Contour for integration of the $\mathbf{G}$ function in the complex plane.

\section{B. Derivation of long time behavior of the linear response function}

From Eq. (68) and Bracewell [39] we get immediately

$$
\widetilde{G}_{n}(\omega)=\frac{1}{\kappa N}\left(1-\frac{\omega_{n}}{\omega_{n}+i \omega}\right)=\frac{i \omega}{\kappa N\left(\omega_{n}+i \omega\right)}
$$

and we need to evaluate the discrete sum

$$
\tilde{G}(\omega)=\sum_{n=0}^{N-1} \frac{i \omega}{\kappa N\left(\omega_{n}+i \omega\right)}
$$

for times $1 / \omega$ larger than $\kappa / 4 \alpha$, so that

$$
\tilde{G}(\omega) \approx \sum_{n=0}^{\infty} \frac{i \omega}{\kappa N\left(\omega_{1} n^{2}+i \omega\right)}=\frac{i}{\kappa N} I\left(\frac{\omega_{1}}{\omega}\right),
$$

where

$$
I(x)=\sum_{n=0}^{\infty} \frac{1}{x n^{2}+i}=\frac{1}{2 i}+J(x)
$$

with

$$
\begin{aligned}
J(x) & =\frac{1}{2} \sum_{-\infty}^{\infty} f(n), \\
f(z) & =\frac{1}{x z^{2}+i} \\
& =\frac{1}{x\left(z-\frac{1-i}{\sqrt{2 x}}\right)\left(z+\frac{1-i}{\sqrt{2 x}}\right)} .
\end{aligned}
$$

To proceed, note that $\int_{C} \operatorname{cotan}(\pi z) f(z)=0$ for a contour $C$ corresponding to $\mathrm{Re}^{i \theta}$ with $R$ a constant that will be sent to infinity and $\theta \in[0,2 \pi[$ (cf. Fig. 7), then by the residue theorem 


$$
0=\sum_{\text {Res }} \operatorname{cotan}(\pi z) f(z)=\frac{1}{\pi} \sum_{n=-\infty}^{\infty} f(n)+\frac{\operatorname{cotan}\left(\pi i^{3 / 2} / \sqrt{x}\right)}{i^{3 / 2} \sqrt{x}} .
$$

So that

$$
J(x)=\frac{i \pi}{2} \frac{\operatorname{cotan}\left(i^{3 / 2} \pi / \sqrt{x}\right)}{\sqrt{i x}}
$$

and from Eq. (77),

$$
\widetilde{G}(\omega)=\frac{1}{2 \kappa N} g\left(\pi i^{3 / 2} \sqrt{\omega / \omega_{1}}\right)
$$

with

$$
g(z)=1+z \operatorname{cotan}(z) \text {. }
$$

The function $g(z)$ is monotonously growing, with limits $g(z) \rightarrow 1$ when $z \rightarrow 0$, and $g(z) \rightarrow \pi z / 2$ when $z \rightarrow \infty$.

Consequently, for finite chains, the long time limit is given by $\widetilde{G}(\omega \rightarrow 0) \sim 1 /(2 \kappa N)$, if $\omega_{1}$ is fixed and finite. This corresponds to a normal diffusive behavior.

However, for very long chains, the transient time to reach this saturaton is extremely long, and corresponds to the regime physically probed by the system. This is seen by taking first the $N \rightarrow \infty$ limit in the above, so that using $\omega_{2}=\omega_{1} N^{1}$ $=(2 \pi)^{2} \alpha / \kappa$,

$$
\tilde{G}(\omega)=\frac{\pi^{2} i^{3 / 2}}{4 \kappa N} \sqrt{\omega / \omega_{1}}=\frac{\pi^{2} i^{3 / 2}}{4 \kappa} \sqrt{\omega / \omega_{2}}=\frac{\pi i^{3 / 2}}{8} \sqrt{\omega /(\alpha \kappa)}
$$

for any $\omega$.

Thus, the Fourier transform of the response function is from Eq. (72),

$$
\tilde{\Gamma}(\omega)=\frac{2 \kappa}{\pi m} \sqrt{\frac{\omega_{2}}{i \omega}}=\frac{4}{m} \sqrt{\frac{\alpha \kappa}{i \omega}},
$$

and from Ref. [8], since the Laplace transform $\hat{\Gamma}$ of $\Gamma$ in the limit of small $\omega$ (long times) behaves as

$$
\hat{\Gamma}(\omega) \sim \widetilde{\Gamma}(-i \omega) \sim \omega^{-1 / 2},
$$

we have for the rms displacement of the observed particle,

$$
\left\langle x^{2}(t)\right\rangle \propto t^{1-1 / 2}=t^{1 / 2}
$$

which is nothing but the subdiffusive regime we observe up until saturation. Thus, our system experimentally, analytically, and numerically verifies the theory for anomalous diffusion in systems with memory derived in Ref. [8], and thus provides an experimental realization of this theory.

\section{CONCLUSION}

Chains of dipoles suspended between two plates, roughen dynamically under the effect of thermal disorder when the dipolar interaction is decreased. This dynamic roughening is intimately related to the scaling of the equilibrium fluctua- tion of these chains. We have studied the dynamic roughening experimentally and theoretically and the equilibrium fluctuations of such chains composed of magnetic holes in a ferrofluid. Their dynamic roughening and fluctuations are shown to behave according to a discrete Rouse model. The rms width of these chains behaves initially as normal diffusion, $W^{2} \propto t$, then it grows with an anomalous exponent, i.e., $W^{2} \propto t^{1 / 2}$ as Fourier modes progressively saturate from the small scales to the large ones. Eventually $W$ reaches an amplitude $W^{2} \sim N$ at large times. We have derived this behavior analytically, including the prefactors, and verified that the experimental data coincide with the theoretical predictions over five decades in the case of the equilibrium fluctuations. The analytic predictions and experiments also coincide with Brownian dynamic simulations taking into account the nonlinearities of the interaction potential, and the interactions beyond nearest neighbors.

Eventually, we have shown that the Markovian description of the entire Brownian worm can be reduced to a nonMarkovian one for a single particle. This description contains a linear response function, with a long time tail decaying as $t^{-\alpha}$ with $\alpha=1 / 2$. Thus, since these chains experimentally exhibit the anomalous diffusion exponent $1 / 2=1$ $-\alpha$, predicted by the theory of diffusion in systems with memory, they constitute a first experimental realization of this theory.

\section{APPENDIX A: DERIVATION OF THE REAL-TIME EXPRESSION OF THE RESPONSE FUNCTION}

We will now perform the inverse time Fourier transform (TFT) from Eq. (87): for that purpose, we introduce a small parameter $\varepsilon$ and write

$$
\Gamma(t)=\lim _{\varepsilon \rightarrow 0} \frac{1}{2 \pi} \frac{2 \kappa}{\pi m} \int_{\omega=-\infty}^{\infty} d \omega e^{i \omega t} \frac{i-1}{\sqrt{2}} \frac{\sqrt{\omega_{2}}}{\sqrt{\omega-i \varepsilon}} .
$$

We define in the complex plane, the square root function $\sqrt{ }$. to have a branch cut along the axis $i x, x>0$, i.e., $\lim _{\delta \rightarrow 0^{+}} \sqrt{i+\delta}=\frac{1+i}{\sqrt{2}}$, and $\lim _{\delta \rightarrow 0^{+}} \sqrt{i-\delta}=\frac{-1-i}{\sqrt{2}}$.

For $t<0$, we close the contour in complex plane by a half-circle in the lower half-plane, and since no singularity is enclosed, $\Gamma(t)=0$ in that case: the response function is causal as desired.

For $t>0$, we get by following the contour in Fig. 8,

$$
\begin{aligned}
\int_{\omega=-\infty}^{\infty} d \omega \frac{e^{i \omega t}}{\sqrt{\omega-i \varepsilon}}= & \int_{x=+\infty}^{0} i d x e^{-x t} \frac{1}{\sqrt{i(x-\varepsilon)+\delta}} \\
& +\int_{x=0}^{+\infty} i d x e^{-x t} \frac{1}{\sqrt{i(x-\varepsilon)-\delta}} \\
\simeq & -2 \frac{\sqrt{2} i}{1+i} \int_{0}^{\infty} \frac{e^{-x t}}{\sqrt{x}} d x=-2 \frac{\sqrt{2} i}{1+i} \sqrt{\frac{\pi}{t}}
\end{aligned}
$$

and thus from Eq. (23),

$$
\Gamma(t)=\Theta(t) \frac{2 \kappa \sqrt{\omega_{2}}}{m \sqrt{\pi^{3} t}}=\Theta(t) \frac{4 \sqrt{\kappa \alpha}}{m \sqrt{\pi t}} .
$$




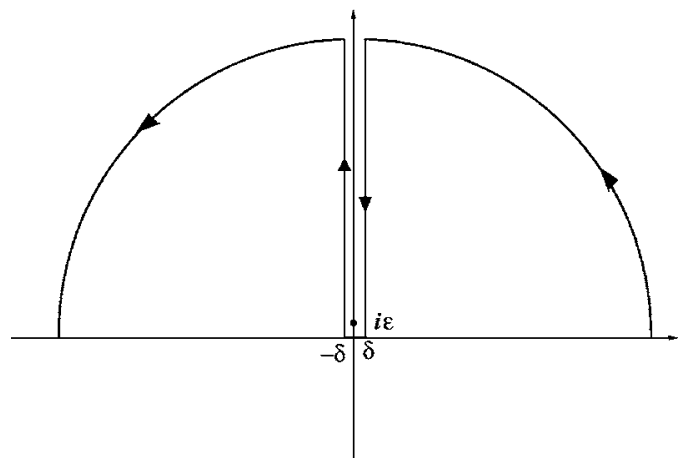

FIG. 8. Contour for the obtention of the linear response function, in the complex plane.

\section{APPENDIX B: AUTOCORRELATION FUNCTION OF THE FLUCTUATING FORCE}

We will here check directly the validity generalized fluctuation dissipation theorem for our specific system with memory, i.e., derive Eq. (75).

We will first use the fluctuation dissipation theorem on the basic set of equations, that led to Eq. (60) for the autocorrelation function of the fluctuating force from the fluid on every particle. Performing a space Fourier transform, Eq. (60) translates for the correlations between the Fourier modes of the fluctuating force, to

$$
\left\langle\zeta_{m}^{\prime}(t) \zeta_{n}^{\prime *}(0)\right\rangle=2 N k_{B} T \delta_{m n} \delta(t) .
$$

We now perform a TFT of $\zeta_{n}^{\prime}(t)$ to obtain from the above and Eq. (70),

$$
\begin{aligned}
\left\langle\widetilde{\zeta}_{m}^{\prime}(\omega) \tilde{\zeta}_{n}^{\prime *}\left(\omega^{\prime}\right)\right\rangle & =\int_{-\infty}^{\infty} d t \int_{-\infty}^{\infty} d t^{\prime}\left\langle\zeta_{m}^{\prime}(t) \zeta_{n}^{\prime *}\left(t^{\prime}\right)\right\rangle e^{-i\left(\omega t-\omega^{\prime} t^{\prime}\right)} \\
& =2 N \kappa k_{B} T \delta_{m n} \int_{-\infty}^{\infty} d t e^{-i\left(\omega-\omega^{\prime}\right) t} \\
& =4 \pi N \kappa k_{B} T \delta_{m n} \delta\left(\omega-\omega^{\prime}\right)
\end{aligned}
$$

We will now express the time autocorrelation function of $F(t)$, the total (chain+fluid) fluctuating force on particle 0 , defined in Eq. (73): using also Eqs. (76) and (77), we obtain

$$
\begin{aligned}
\left\langle F(t) F^{*}(0)\right\rangle= & \frac{1}{(2 \pi)^{2}} \int_{-\infty}^{\infty} d \omega \int_{-\infty}^{\infty} d \omega^{\prime} \frac{4 \kappa^{2}}{\pi^{2}} \frac{\omega_{1}}{\omega} \frac{\omega^{2}}{\kappa^{2}} \frac{e^{i \omega t}}{N^{2}} \\
& \times \sum_{m n} \frac{\left\langle\widetilde{\zeta}_{m}^{\prime}(\omega) \tilde{\zeta}_{n}^{\prime *}\left(\omega^{\prime}\right)\right\rangle}{\left(\omega_{m}+i \omega\right)\left(\omega_{n}-i \omega\right)} \\
= & \frac{8 \kappa k_{B} T / \pi^{2}}{2 \pi} \int_{-\infty}^{\infty} d \omega s(\omega) e^{i \omega t}
\end{aligned}
$$

with

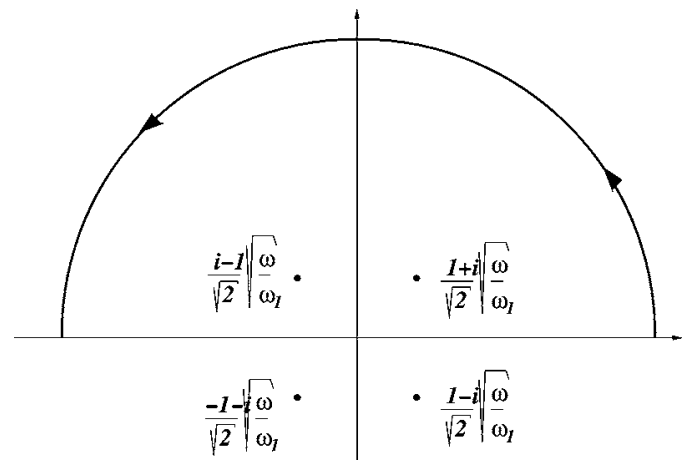

FIG. 9. Contour in the complex plane for the integration of the time autocorrelation of the fluctuating force.

$$
\begin{aligned}
s(\omega) & =\frac{\omega_{1} \omega}{N} \sum_{n} \frac{1}{\omega_{n}^{2}+\omega^{2}} \\
& \simeq \frac{\omega_{1} \omega}{N} \sum_{n} \frac{1}{\omega_{1}^{2} n^{4}+\omega^{2}} \\
& \simeq \frac{1}{2} \frac{\omega}{\omega_{1}} \int_{-\infty}^{\infty} \frac{d x}{x^{4}+\frac{\omega^{2}}{\omega_{1}^{2}}} \\
& =\frac{\pi i \omega}{\sqrt{2} \omega_{1}}\left(\frac{1}{-i(i-1)}+\frac{1}{i(i+1)}\right)\left(\frac{\omega_{1}}{\omega}\right)^{3 / 2} \\
& =\frac{\pi}{\sqrt{2}}\left(\frac{\omega_{1}}{\omega}\right)^{1 / 2}
\end{aligned}
$$

(see Fig. 9 for the contour adopted in application of the residue theorem). Thus, introducing the notation

$$
\left\langle F(t) F^{*}(0)\right\rangle=g(t)
$$

we obtain from Eqs. (87) and (B4)-(B6),

$$
\tilde{g}(\omega)=\frac{8 \kappa k_{B} T}{\pi \sqrt{2}} \sqrt{\frac{\omega_{1}}{\omega}}=2 m k_{B} T\left[\tilde{\Gamma}(\omega)+\widetilde{\Gamma}^{*}(\omega)\right]
$$

which means that

$$
\left\langle F(t) F^{*}(0)\right\rangle=2 m k_{B} T \Gamma(|t|),
$$

where we have used the fact that since the response function is real,

$$
\widetilde{\Gamma}^{*}(\omega)=\frac{1}{2 \pi} \int_{-\infty}^{\infty} \Gamma(t) e^{i \omega t} d t=\frac{1}{2 \pi} \int_{-\infty}^{\infty} \Gamma(-t) e^{-i \omega t} d t
$$

and since it is causal, $\Gamma(t)+\Gamma(-t)=\Gamma(|t|)$. Equation (B8) corresponds to the fluctuation dissipation theorem for a system with memory, as presented in Ref. [8]. 
[1] A. T. Skjeltorp, Phys. Rev. Lett. 51, 2306 (1983).

[2] R. Toussaint, J. Akselvoll, G. Helgesen, A. T. Skjeltorp, and E. G. Flekkøy, Phys. Rev. E 69, 011407 (2004).

[3] A. Skjeltorp and G. Helgesen, Physica A 176, 37 (1991).

[4] J. Ugelstad and P. Mork, Adv. Colloid Interface Sci. 13, 101 (1980).

[5] R. Toussaint, G. Helgesen, and E. G. Flekkøy, Phys. Rev. Lett. 93, 108304 (2004).

[6] A. Y. Grosberg and A. R. Khokhlov, Statistical Physics of Macromolecules (AIP Press, New York, 1994).

[7] S. F. Edwards and D. R. Wilkinson, Proc. R. Soc. London, Ser. A 381, 17 (1982).

[8] R. Morgado, F. A. Oliveira, G. G. Batrouni, and A. Hansen, Phys. Rev. Lett. 89, 100601 (2002).

[9] E. M. Furst and A. P. Gast, Phys. Rev. E 62, 6916 (2000).

[10] E. M. Furst and A. P. Gast, Phys. Rev. E 58, 3372 (1998).

[11] S. Cutillas and J. Liu, Phys. Rev. E 64, 011506 (2001).

[12] A. S. Silva, R. Bond, F. Plouraboué, and D. Wirtz, Phys. Rev. E 54, 5502 (1996).

[13] M. R. Jolly, J. W. Bender, and J. D. Carlson, J. Intell. Mater. Syst. Struct. 10, 5 (1999).

[14] S. F. Dyke, B. F. Spencer, M. K. Sain, and J. D. Carlson, Smart Mater. Struct. 7, 693 (1998).

[15] Proceedings of the 5th International Conference on Electrorheological Fluids, Magneto-rheological Suspensions and Associated Technology, edited by W. A. Bullough (World Scientific, Singapore, 1996).

[16] O. Ashour, C. Rogers, and W. Kordonsky, J. Intell. Mater. Syst. Struct. 7, 123 (1996).

[17] J. A. Stangroom, Phys. Technol. 14, 290 (1983).

[18] T. C. Halsey and W. Toor, J. Stat. Phys. 61, 1257 (1990).

[19] T. C. Halsey and W. Toor, Phys. Rev. Lett. 65, 2820 (1990).

[20] T. C. Halsey, J. Colloid Interface Sci. 156, 335 (1993).

[21] J. E. Martin, J. Odinek, and T. C. Halsey, Phys. Rev. Lett. 69, 1524 (1992).

[22] J. E. Martin, K. M. Hill, and C. P. Tigges, Phys. Rev. E 59,
5676 (1999).

[23] M. Fermigier and A. P. Gast, J. Colloid Interface Sci. 154, 522 (1992).

[24] G. Helgesen, A. T. Skjeltorp, P. M. Mors, R. Botet, and R. Jullien, Phys. Rev. Lett. 61, 1736 (1988).

[25] S. Fraden, A. J. Hurd, and R. B. Meyer, Phys. Rev. Lett. 63, 2373 (1989).

[26] J. E. Martin, J. Odinek, T. C. Halsey, and R. Kamien, Phys. Rev. E 57, 756 (1998).

[27] J. Liu, E. M. Lawrence, A. Wu, M. L. Ivey, G. A. Flores, K. Javier, J. Bibette, and J. Richard, Phys. Rev. Lett. 74, 2828 (1995).

[28] J. Cernak, G. Helgesen, and A. T. Skjeltorp, Phys. Rev. E 70, 031504 (2004).

[29] Type EMG 909, produced by Ferrofluidics Corporation, 40 Simon St., Nashua, NH 03061.

[30] B. Bleaney and B. Bleaney, Electricity and Magnetism (Oxford University Press, Oxford, 1978).

[31] P. G. De Gennes and P. Pincus, Phys. Kondens. Mater. 11, 1970 (1970).

[32] E. G. Flekkøy and D. H. Rothman, Phys. Rev. E 53, 1622 (1996).

[33] E. G. Flekkøy and D. H. Rothman, Phys. Rev. Lett. 75, 260 (1995).

[34] F. Reif, Fundamentals of Statistical and Thermal Physics (McGraw-Hill, Singapore, 1965).

[35] F. Family and T. Vicsek, J. Phys. A 18, L75 (1985).

[36] L. P. Faucheux and A. J. Libchaber, Phys. Rev. E 49, 5158 (1994).

[37] M. Sahimi, Flow and Transport in Porous Media and Fractured Rocks (Wiley-VCH, New York, 1995).

[38] W. H. Press et al., Numerical Recipes (Cambridge University Press, New York, 1992).

[39] R. N. Bracewell, The Fourier Tranform and its Applications, 3rd ed. (McGraw-Hill, Singapore, 2000). 